Topics

\title{
Objectives, Outlines, and Preparation for the Resist Tubule Space Experiment to Understand the Mechanism of Gravity Resistance in Plants
}

\author{
By Takayuki Hoson ${ }^{1}$, Haruhiko AKAMATSU ${ }^{1)}$, Kouichi SoGA ${ }^{1)}$, Kazuyuki WAKABAYASHI ${ }^{1)}$, \\ Hirofumi HASHImOto ${ }^{2)}$, Masamichi Yamashita ${ }^{2)}$, Katsuya HaseGaWA ${ }^{2)}$, Sachiko YANO ${ }^{3)}$, \\ Katsunori OMORI $^{3)}$, Noriaki IsHIOKA ${ }^{3)}$, Shohei MATSUMOTO ${ }^{4)}$, Haruo KASAHARA ${ }^{4)}$, \\ Toru SHIMAZU ${ }^{5)}$, Shoji A. BABA ${ }^{6)}$ and Takashi HASHIMOTO ${ }^{7)}$ \\ ${ }^{1)}$ Department of Biology, Osaka City University, Osaka, Japan \\ 2) Japan Aerospace Exploration Agency, Sagamihara, Japan \\ ${ }^{3)}$ Japan Aerospace Exploration Agency, Tsukuba, Japan \\ 4) Japan Manned Space Systems, Tokyo, Japan \\ ${ }^{5)}$ Japan Space Forum, Tokyo, Japan \\ ${ }^{6}$ Ochanomizu University, Tokyo, Japan \\ ${ }^{7)}$ Nara Institute of Science and Technology, Ikoma, Japan
}

(Received June 27th, 2011)

\begin{abstract}
Gravity resistance is a principal graviresponse in plants. In resistance to hypergravity, the gravity signal may be perceived by the mechanoreceptors located on the plasma membrane, and then transformed and transduced via the structural continuum or physiological continuity of cortical microtubules-plasma membrane-cell wall, leading to an increase in the cell wall rigidity as the final response. The Resist Tubule experiment, which will be conducted in the Kibo Module on the International Space Station, aims to confirm that this hypothesis is applicable to resistance to $1 G$ gravity. There are two major objectives in the Resist Tubule experiment. One is to quantify the contributions of cortical microtubules to gravity resistance using Arabidopsis tubulin mutants with different degrees of defects. Another objective is to analyze the modifications to dynamics of cortical microtubules and membrane rafts under microgravity conditions on-site by observing green fluorescent protein (GFP)-expressing Arabidopsis lines with the fluorescence microscope in the Kibo. We have selected suitable mutants, developed necessary hardware, and fixed operation procedure for the experiment.
\end{abstract}

Key Words: Arabidopsis, Gravity Resistance, Microtubule, Plant, Resist Tubule

\section{Introduction}

Mechanical resistance to the gravitational force is a principal graviresponse in plants, distinct from and comparable to gravitropism, and has been termed 'gravity resistance' ${ }^{1)}$. The development of gravity resistance has played an important role in the transition of plant ancestors from an aquatic environment to a terrestrial environment about 450 million years ago and in the consequent establishment of land plants ${ }^{2)}$. Nevertheless, the presence of this graviresponse has not been properly recognized for long, and only limited information has been obtained for the mechanism of gravity resistance. We have examined its mechanism using hypergravity conditions produced by centrifugation ${ }^{1,3)}$. As a result, we have clarified the outline of the sequence of events in gravity resistance to hypergravity. The gravity signal may be perceived by the mechanoreceptors located on the plasma membrane in resistance to hypergravity. The perceived signal may be transformed and transduced via the structural continuum or physiological continuity of cortical microtubules and the plasma membrane, and then induce the expression of diverse genes and influence the formation and structure of various cellular components. The modifications of the cell wall environment, in combination with the changes in the levels and the molecular size of the cell wall constituents, may increase the cell wall rigidity as the final response. However, it is uncertain whether the hypothesis formulated is applicable to gravity resistance to $1 G$ gravity, as to the resistance to hypergravity. To clarify this point, we will conduct a space experiment termed Resist Tubule in the Kibo Module on the International Space Station. In the present article, we describe the objectives and outlines of the Resist Tubule space experiment, as well as the preparation for the experiment.

\section{Objectives of the Resist Tubule Experiment}

There are two major objectives in the Resist Tubule experiment. One is to quantify the contributions of cortical microtubules to gravity resistance using Arabidopsis tubulin mutants with different degrees of defects in growth phenotypes. Another objective is to analyze the modifications to dynamics of cortical microtubules and membrane rafts under microgravity conditions on-site by observing GFP-expressing Arabidopsis lines with the fluorescence microscope in the Kibo Module.

\subsection{Quantitative characteristics of gravity resistance}

Arabidopsis mutants defective in formation of cortical microtubules or the plasma membrane are unable to form the normal cell wall, and therefore, they show disordered growth 
pattern, such as dwarfism and helical growth, on earth. The unfavorable phenotypes are intensified and the mutants show a low viability under hypergravity conditions. However, it is expected that the defects of such mutants are rescued and they can grow and develop more or less normally under microgravity in space, where formation of the tough cell wall is not required. In the Resist Wall experiment, which was conducted in the European Modular Cultivation System (EMCS) in the Columbus Module on March - May, 2008, we intended to examine this hypothesis by cultivating a tubulin mutant, tua6, as well as the wild-type Columbia under $1 G$ and microgravity conditions up to reproductive stage and comparing phenotypes on growth and development using video images taken with an automated acquisition system. Unfortunately, we faced great difficulties in initial watering because of serious anomalies of water supply system in EMCS, probably due to incorrect connections between the life support system and water reservoirs ${ }^{4)}$. Thus, no plants developed until the expected developmental stage ${ }^{5}$.

In the Resist Tubule experiment, we will challenge again to confirm the above-mentioned hypothesis. At the same time, we will quantify the contributions of cortical microtubules to gravity resistance using tubulin mutants with different degrees of defects in growth phenotypes. There is a good correlation between the degree of defects on formation of cortical microtubules and the capacity of gravity resistance under hypergravity conditions. Thus, tubulin mutants may become hypersensitive to the gravitational force, dependent on the degree of defects. Also, we have shown that parameters on gravity resistance may vary in proportion to the logarithm of the magnitude of gravity over microgravity and hypergravity range ${ }^{1)}$. We intend to confirm such a dose-response relationship by the Resist Tubule experiment.

\subsection{Dynamics of cellular components}

The formation and the structure of cortical microtubules and membrane rafts are greatly influenced by the gravitational force. For instance, hypergravity increased transiently the expression of $\gamma$-tubulin and katanin genes ${ }^{6,7)}$ as well as that of $\alpha$ - and $\beta$-tubulin genes, thereby inducing prompt reorientation of cortical microtubules from transverse to longitudinal directions in epidermal cells of stem organs ${ }^{8}$. So far, there were no studies on the formation or the structure of these cellular components in plants under microgravity conditions. In particular, it is meaningful to analyze the modifications to their dynamics under microgravity conditions on-site in real time by observing GFP-expressing lines with the fluorescence microscope in the Kibo Module.

\section{Outlines of the Resist Tubule Experiment}

The Resist Tubule experiment consists of three runs. We aim to analyze the modifications to dynamics of cortical microtubules and membrane rafts under microgravity conditions in space in Runs 1 and 2. On the other hand, Run 3 is for quantifying the contributions of cortical microtubules to gravity resistance (Fig. 1).

\subsection{Quantitative characteristics of gravity resistance}

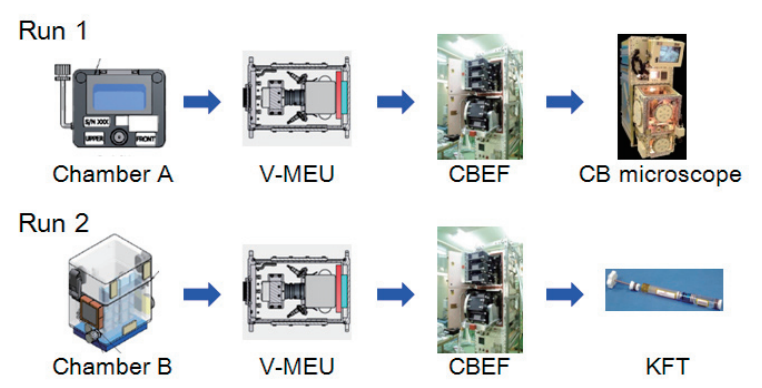

Run 3

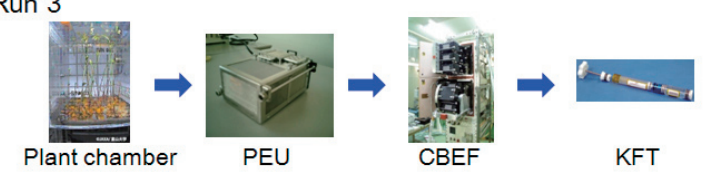

Fig. 1. On-board operations in the Resist Tubule experiment. The experiment consists of three runs.

In Run 3, dry seeds of tubulin mutants with different degrees of defects in growth phenotypes, as well as wild-type Columbia and 3-hydroxy-3-methylglutaryl-Coenzyme A reductase (HMG) mutant, are sown in the Plant Chamber and set to the Plant Experiment Unit (PEU), developed for the Space Seed experiment (PI, Prof. S. Kamisaka). Two sets of PEUs are prepared: one is for microgravity and the other for flight $1 G$ control. These PEUs are stowed and launched at ambient temperature, and then set into the Cell Biology Experiment Facility (CBEF) in the Kibo Module in orbit. Plants are germinated and grown at $23.5^{\circ} \mathrm{C}$ in the light for 32 days. Cultivation starts by water supply and plants are supplied with nutrients and water automatically thereafter. Plants are regularly observed with a video camera by automated system equipped in the CBEF. Video images are down-linked to earth in real time or within a minimum delay. When inflorescences of wild-type and mutants fully develop, the PEUs are taken out of the CBEF. Developed inflorescences are then put into the Kennedy Space Center Fixation Tube (KFT) and fixed with RNAlater ${ }^{\circledR}$ solution (Ambion). KFTs are kept at $2^{\circ} \mathrm{C}$ for several days and then kept frozen in orbit and during recovery to earth. An identical experiment in terms of materials, hardware, and procedures are carried out in the laboratory of PI as the ground control.

Two types of data are obtained in Run 3 of the Resist Tubule experiment: video images and plant materials collected. We quantify parameters on growth and development, such as the length and the shape of hypocotyls, roots, flower stalks and siliques, and the size and the number of rosette leaves, cauline leaves and flowers from down-linked video images. Based on measured data, we compare differences in these phenotypes among Columbia and different tubulin mutants. Also, we measure the mechanical properties of the cell walls of thawed inflorescences, which had been fixed with RNAlater ${ }^{\circledR}$ solution in KFTs in orbit and collected to earth, with a tensile tester. We then extract total RNA from buds, leaves, and young flower stalks of collected plant materials and synthesize the first-strand complementary DNA (cDNA) 
using commercial preparation kits. The expressions of genes encoding tubulins, microtubule-associated proteins (MAPs), HMG, and xyloglucan endo-transglucosylase/hydrolases are analyzed with the real-time polymerase chain reaction (PCR) method. The cell wall constituents are finally collected from the remaining materials, and then the levels of the cell wall polysaccharides and the molecular size of xyloglucans are measured colorimetrically and with a gel filtration column on high performance liquid chromatography (HPLC), respectively.

\subsection{Dynamics of cellular components}

In Run 1, dry seeds of GFP-expressing Arabidopsis lines are sown in the Cultivation/Observation Chamber (Chamber A) and set to the Video-Measurement Unit (V-MEU). Two sets of V-MEUs are prepared: one is for microgravity and the other for flight $1 G$ control. These V-MEUs are stowed at ambient temperature, and then set into the CBEF in the Kibo Module in orbit. Seeds are germinated and seedlings are grown at $25^{\circ} \mathrm{C}$ in the dark. After three days, the V-MEUs are taken out of the CBEF and transported into the Clean Bench (CB). Cortical microtubules and membrane rafts in epidermal cells of hypocotyls, labeled with GFP, are observed with the fluorescence microscope in the $\mathrm{CB}$ (CB microscope). Micrographs are saved and down-linked to earth in real time or within a minimum delay.

In Run 2, dry seeds of Columbia are sown in the Chamber $\mathrm{B}$ and set to the V-MEU, and then cultivated as mentioned above. After three days, the V-MEUs are taken out of the $\mathrm{CBEF}$, and then developed seedlings are put into the KFTs and fixed with a mixture of $0.5 \%$ glutaraldehyde and $1.5 \%$ formaldehyde. KFTs are kept at $2^{\circ} \mathrm{C}$ in orbit and during recovery to earth. Fixed materials of this set are used for observation of modifications by microgravity of the formation and structure of various cellular components, including those not used for GFP-labeling. Thus, this experiment is important not only as a backup of GFP observation in orbit (Run 1) but for obtaining a wide range of information on modifications to various cellular components and the mechanism of gravity resistance.

\section{Preparation for the Resist Tubule Experiment}

We have selected suitable Arabidopsis mutants (lines), developed necessary hardware, and fixed operation procedure for the Resist Tubule experiment.

\subsection{Quantitative characteristics of gravity resistance}

A number of amino acid substitution mutants in $\alpha$ - or $\beta$ tubulins have been isolated in Arabidopsis ${ }^{9}$, from which we selected suitable mutants for the Resist Tubule experiment. Hypocotyls of tubulin mutants were shorter and thicker than the wild-type even at $1 G$, and hypergravity further suppressed elongation and stimulated expansion. The degree of such changes was clearly smaller in tubulin mutants ${ }^{10}$. Hypocotyls of tubulin mutants also showed either left-handed or right-handed helical growth at $1 G$, and the degree of twisting phenotype was intensified under hypergravity conditions. Hypergravity induced reorientation of cortical microtubules
A

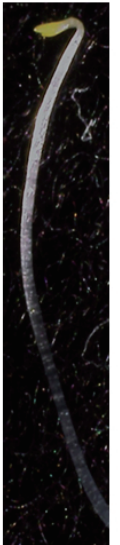

Wild type

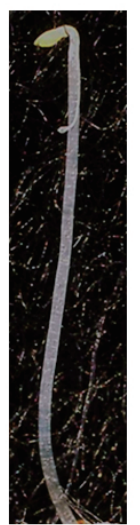

SPR2-GFP

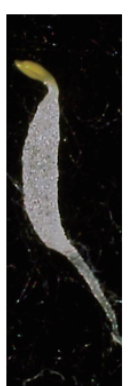

GFP-MAP4
B

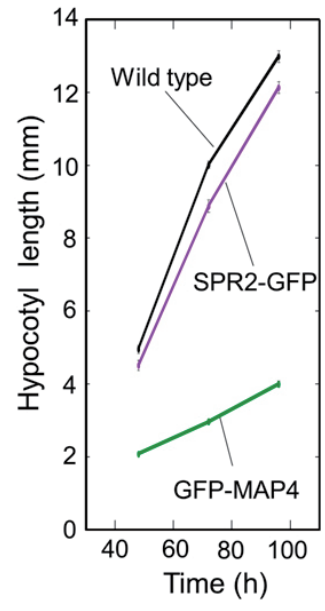

Fig. 2. Growth of GFP-expressing Arabidopsis lines. Seedlings of SPR2-GFP and GFP-MAP4 as well as the wild-type Columbia were grown at $25^{\circ} \mathrm{C}$ in the dark for 3 days (A), and the length of hypocotyls was measured $(B)$. Values are means $\pm S E(n=20)$.

from transverse to longitudinal directions in epidermal cells of wild-type hypocotyls. In tubulin mutants, the percentage of cells with longitudinal microtubules was high even at $1 G$, and it was further increased by hypergravity. All these phenotypes were clearer in tua6 (A281T) mutant than in tua3 (D205N) or tua4 $(\mathrm{S} 178 \Delta)$ mutant. Thus, we compare capacity of gravity resistance of tua 6 with that of tua 3 or tua 4 under microgravity conditions in space. Also, there still remains a possibility that we will use other sets of tubulin mutants with different degrees of defects in microtubule organization.

\subsection{Dynamics of cellular components}

We have produced three types of GFP-expressing lines in Arabidopsis and selected suitable ones for the Resist Tubule

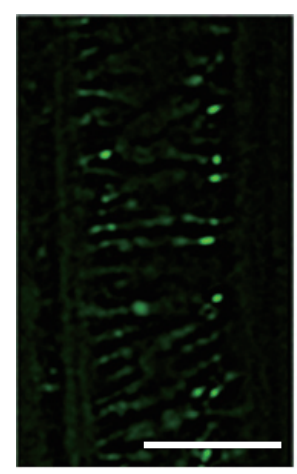

SPR2-GFP

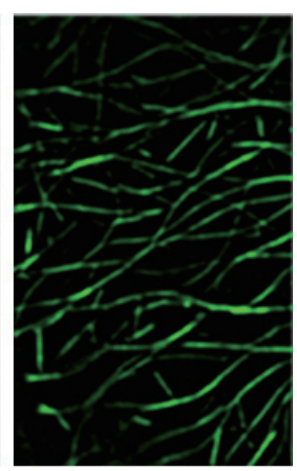

GFP-MAP4
Fig. 3. Fluorescence micrographs of cortical microtubules in epidermal cells of Arabidopsis hypocotyls. Seedlings of SPR2-GFP and GFP-MAP4 were grown in the dark for 3 days, and then GFP fluorescence of epidermal cells was observed with a fluorescence microscope. The bar denotes $10 \mu \mathrm{m}$. 


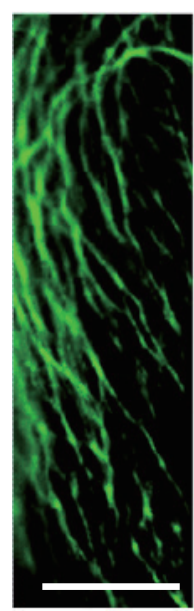

GFP

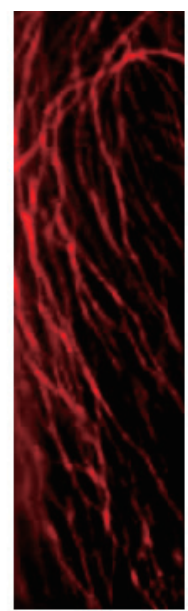

Antibody

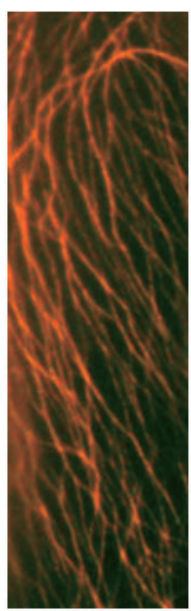

Merge
Fig. 4. Fluorescence micrographs of cortical microtubules in epidermal cells of GFP-MAP4. Seedlings of GFP-MAP4 were grown as in Fig. 2, and their hypocotyls were treated with $\alpha$-tubulin antibodies and then with Cy3-labeled secondary antibodies. Fluorescence due to GFP or antibodies was observed with a fluorescence microscope. Right, images of GFP and antibody staining were merged. The bar denotes $10 \mu \mathrm{m}$.

experiment. In the first group, GFP gene is expressed under the transcriptional control of tubulin, MAPs, or raft protein promoters. Such GFP reporter lines are useful for examining changes in gene expression, and their growth phenotypes are normal, but they are not applicable to observation of dynamics of cellular components. In the second lines, tubulin, MAPs, or raft protein genes fused with GFP genes are expressed under the control of the endogenous promoters. They are useful for both gene expression analysis and observation of cortical microtubules and membrane rafts, but in general fluorescence due to GFP is not strong enough. In the third group, GFP proteins fused with N-terminal region of tubulin, MAPs, or raft proteins are produced under the $35 \mathrm{~S}$ promoter of Cauliflower mosaic virus. They show strong fluorescence, but their growth phenotype is often modified. We selected totally four lines from these three groups for the Resist Tubule experiment. Seedlings of SPIRAL2(SPR2)pro::SPR2-GFP and 35Spro::GFP-MAP4 lines were grown and elongation growth of their hypocotyls was measured (Fig. 2). Hypocotyls of SPR2-GFP elongated at almost the same rate as the wild-type, whereas elongation growth of GFP-MAP4 was strongly suppressed. In GFP-MAP4 hypocotyls, lateral expansion was instead promoted. On the other hand, epidermal cells of SPR2-GFP hypocotyls showed weak, whereas those of GFP-MAP4 strong fluorescence due to GFP (Fig. 3). We have decided to use both lines for observing cortical microtubules in orbit. Fluorescence microscopic analysis revealed that SPR2-GFP and GFP-MAP4 fluorescence aligned along filamentous structures in the cortical region of epidermal cells, which were also stained with tubulin antibodies (Fig. 4). The result indicates that these GFP-expressing lines are useful for the Resist Tubule experiment.
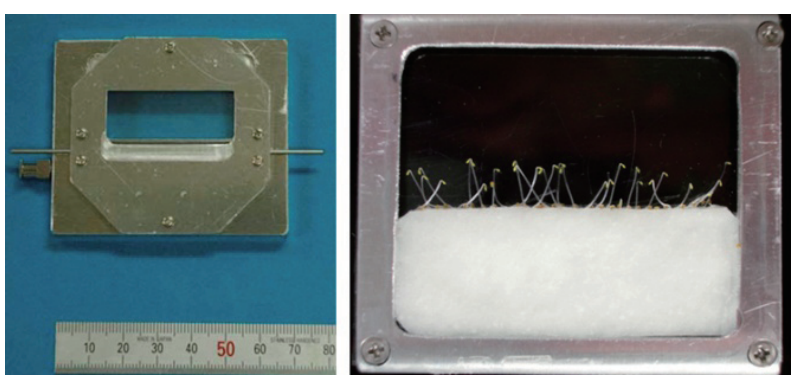

Fig. 5. The prototype of Cultivation/Observation Chamber (Chamber A) developed for analysis of modifications to dynamics of cellular components in orbit (Run 1).

One major difficulty in analyzing the modifications to dynamics of cortical microtubules and membrane rafts under microgravity conditions on-site is in the step to prepare glass slide sets for microscopic observation. This process is of routine on the ground, but it is very difficult to be conducted under microgravity conditions in orbit. We, therefore, newly designed the Chamber $\mathrm{A}$, in which seedlings are cultivated and then directly used for observation, without transporting and preparing of another slide sets (Fig. 5). The usefulness of the Chamber A for the protocol of the Resist Tubule experiment was checked and confirmed by preliminary experiments on the ground.

Another serious problem for observing GFP-labeling in orbit is that the CB microscope is original 'classical' type fluorescence microscope and has not been renewed for more than 20 years after it was developed. We intend to improve its capacity as much as possible by attaching a high-definition EM-CCD camera, type EM246 (Raptor Photonics), specially modified for the Japan Aerospace Exploration Agency (JAXA), and by using the image-processing software developed by JAXA. When epidermal cells of GFP-MAP4 hypocotyls were observed with an improved microscopic system, filamentous fluorescence corresponding to cortical microtubules, was observed, indicating the effectiveness of this improvement.

\section{Conclusions and Perspectives}

Gravity resistance is the major graviresponse in plants and has supported the transition of plant ancestors from an aquatic environment to a terrestrial environment and their evolution as land plants. The Resist Tubule experiment will greatly deepen our knowledge of its mechanism, thereby clarifying the processes of plant evolution. The experiment will also advance our understanding of the mechanisms of other environmental responses in plants, because the processes by which plants respond to other signals are often common to those of gravity resistance. Plants are forced to use more than a half of energy, fixed by photosynthesis, for producing the touch cell wall to resist the gravitational force on earth. Thus, gravity resistance is the process directly related to plant 
production. The Resist Tubule experiment may contribute to efficient plant production indispensable for human life not only in space but also on earth.

\section{Acknowledgments}

We thank many colleagues involved in the Resist Tubule experiment for their great efforts and cooperation. Supports from JAXA are also greatly appreciated.

\section{References}

1) Hoson, T. and Soga, K.: New Aspects of Gravity Responses in Plant Cells, Int. Rev. Cytol., 229 (2003), pp. 209-244.

2) Hoson, T.: The Mechanism and Significance of Gravity Resistance in Plants, J. Gravit. Physiol., 13 (2006), pp. 97-100.

3) Hoson, T., Saito, Y., Soga, K. and Wakabayashi, K.: Signal Perception, Transduction, and Response in Gravity Resistance. Another Graviresponse in Plants, Adv. Space Res., 36 (2005), pp. 1196-1202.

4) Kamada, M., Omori, K., Yokoyama, R., Nishitani, K., Hoson, T., Shimazu, T. and Ishioka, N.: Preparation and Outline of Space-Based Studies on Gravity Responses and Cell Wall Formation in Plants, Biol. Sci. Space, 23 (2009), pp. 115-120.
5) Hoson, T., Matsumoto, S., Soga, K., Wakabayashi, K., Hashimoto, T., Sonobe, S., Muranaka, T., Kamisaka, S., Kamada, M., Omori, K., Ishioka, N. and Shimazu, T.: Growth and Cell Wall Properties in Hypocotyls of Arabidopsis tua6 Mutant under Microgravity Conditions in Space, Biol. Sci. Space, 23 (2009), pp. 71-76.

6) Soga, K., Kotake, T., Wakabayashi, K., Kamisaka, S. and Hoson, T.: Transient Increase in the Transcript Levels of $\gamma$-Tubulin Complex Genes during Reorientation of Cortical Microtubules by Gravity in Azuki Bean (Vigna angularis) Epicotyls, J. Plant Res., 121 (2008), pp. 493-498.

7) Soga, K., Kotake, T., Wakabayashi, K., Kamisaka, S. and Hoson, T.: The Transcript Level of Katanin Gene is Increased Transiently in Response to Changes in Gravitational Conditions in Azuki Bean Epicotyls, Biol. Sci. Space, 23 (2009), pp. 23-28.

8) Soga, K., Wakabayashi, K., Kamisaka, S. and Hoson, T.: Hypergravity Induces Reorientation of Cortical Microtubules and Modifies Growth Anisotropy in Azuki Bean Epicotyls, Planta, 224 (2006), pp. 1485-1494

9) Hashimoto, T.: Molecular Genetics Aanalysis of Left-Right Handedness in Plants, Philos. Trans. Royal Soc. London B. Biol. Sci., 357 (2002), pp. 799-808.

10) Matsumoto, S., Kumasaki, S., Soga, K., Wakabayashi, K., Hashimoto, T. and Hoson, T.: Gravity-Induced Modifications to Development in Hypocotyls of Arabidopsis Tubulin Mutants, Plant Physiol., 152 (2010), pp. 918-926. 